\title{
Aspectos afectivos de las actitudes lingüísticas de estudiantes universitarios *
}

\author{
MÓNICA CHAMORRO MEJÍA ** \\ KAREN LÓPEZ GIL **
}

Recepción: 5 de octubre de 2019

Aprobación: 20 de diciembre de 2019

Forma de citar este artículo: Chamorro, M \& López, K. (2020). Aspectos afectivos de las actitudes lingüísticas de estudiantes universitarios. Cuadernos de Lingüística Hispánica, (35), 37-56.

https://doi.org/ 10.19053/0121053X.n35.2020.10142

* Artículo de investigación derivado del proyecto "Estudio de actitudes lingüísticas de los estudiantes foráneos de primer semestre de la Pontificia Universidad Javeriana Cali hacia el dialecto vallecaucano urbano de Cali y el español académico estándar”, aprobado y financiado en la convocatoria Capital Semilla 2017 de la Pontificia Universidad Javeriana Cali, código 297.

** Mónica Chamorro Mejía, doctora en Lingüística Aplicada de la Universidad de Alcalá de Henares, España. Docente e investigadora del Dpto. de Comunicación y Lenguaje de la Pontificia Universidad Javeriana, seccional Cali; integrante del grupo de investigación "Procesos y Medios de Comunicación" en la línea "Estudios del lenguaje". Correo electrónico: monica. chamorro@javerianacali.edu.co B https://orcid.org/0000-0003-4953-4591

*** Karen López Gil, doctora en Educación de la UNED, España; magíster en Lingüística y Español de la Universidad del Valle, Colombia; docente e investigadora del Dpto. de Comunicación y Lenguaje de la Pontificia Universidad Javeriana, seccional Cali; integrante del grupo de investigación "Procesos y Medios de Comunicación" en la línea "Estudios del lenguaje". Correo electrónico: karenlopez@javerianacali.edu.co $\oplus$ https://orcid.org/0000-0001-9826-0799 


\section{Resumen}

Este artículo describe los aspectos afectivos de las actitudes lingüísticas de estudiantes de diferentes zonas geolectales de Colombia que ingresan a una universidad de la ciudad de Cali. Esta ciudad se caracteriza por su pluralidad cultural y dialectal, en parte debido a las migraciones forzadas desde zonas marcadas por el conflicto armado. Además, en años recientes, el Estado colombiano ha impulsado el ingreso a las universidades de jóvenes estudiantes académicamente sobresalientes, provenientes en su mayoría de zonas rurales. La diversidad de usos de la lengua que los estudiantes han construido en sus contextos de origen puede entrar en tensión con las dinámicas del ámbito universitario. A partir de una perspectiva mentalista en la que priman los fenómenos internos susceptibles de autoobservación, buscamos extrapolar los aspectos afectivos de las actitudes lingüísticas de la población estudiada a través de un matchedguise y un cuestionario, instrumentos que indagan por las percepciones respecto a cinco dialectos que se encuentran con frecuencia en la universidad. Los resultados evidencian valoraciones distintas de los dialectos y una consideración del dialecto bogotano como central, prestigioso y estándar, asociado fuertemente a las formas de oralidad académica.

Palabras clave: actitudes lingüísticas; español estándar; oralidad; dialectos.

\section{Affective Features of Linguistic Attitudes of College Students}

\section{Abstract}

This paper describes the affective features of the linguistic attitudes of students from different geolectal areas of Colombia who attend to a university in Cali. This city is characterized by its cultural and dialectal plurality, partly due to forced migrations from armed conflict areas. In addition, in recent years, the Colombian State has boosted the access of top students to the university, mostly from rural areas. The diversity of uses of the language that students have built in their home contexts could get in tension with the dynamics of the college environment. From a mentalist perspective in which internal phenomena susceptible of self-observation prevail, it seeks to extrapolate the affective features of the linguistic attitudes of the population studied through a matched-guise and a questionnaire, instruments that inquire perceptions regarding five dialects very frequent used in the university. The results show different assessments of the dialects and an opinion on the Bogota accent as central, prestigious and standard, strongly associated with forms of academic orality.

Keywords: linguistic attitudes; standard Spanish; orality; dialects. 


\section{Aspects affectifs des attitudes linguistiques des étudiants universitaires}

\section{Résumé}

Cet article décrit les aspects affectifs des attitudes linguistiques des étudiants de différentes régions géolectales de Colombie, qui commencent leurs études dans une université quelconque de la ville de Cali. Cette ville est connue par sa pluralité culturelle et dialectale, due, en quelque sort, aux migrations forcées et successives en provenance de zones touchées par des conflits armés. En outre, au cours des dernières années, l'État colombien a favorisé l'entrée de jeunes étudiants, en provenance notamment des zones rurales, avec de très hautes performances académiques, à l'université, La diversité d'usages de la langue auxquels les étudiants ont donné vie dans leur contexte d'origine peut entrer en conflit avec la dynamique du monde universitaire. Dans une perspective mentaliste, où des phénomènes internes susceptibles de s'auto-observer sont privilégiés, nous cherchons à extrapoler les aspects affectifs des attitudes linguistiques de la population objet d'étude, à travers une démarche d'accouplement et un questionnaire, outils qui cherchent à établir les perceptions concernant cinq dialectes que l'on retrouve assez souvent chez les étudiants à l'université. Les résultats montrent évaluations différentes des dialectes et une prise en compte du dialecte de Bogota, associé étroitement aux formes d'oralité académique, comme étant le plus accepté, prestigieux et standard.

Mots-clés: attitudes linguistiques; espagnol standard; oralité; dialectes.

\section{Aspectos afetivos das atitudes linguísticas de alunos universitários}

\section{Resumo}

Este artigo descreve os aspectos afetivos das atitudes lingüísticas de estudantes de diferentes áreas geográficas da Colômbia que ingressam em uma universidade na cidade de Cali. Esta cidade é caracterizada por sua pluralidade cultural e dialetal, em parte devido a migrações forçadas de áreas marcadas por conflitos armados. Além disso, nos últimos anos, o Estado colombiano aumentou a entrada de jovens estudantes academicamente destacados nas universidades, principalmente das áreas rurais. A diversidade de usos da linguagem que os alunos construíram em seus contextos domésticos pode entrar em tensão com a dinâmica do ambiente universitário. De uma perspectiva mentalista em que prevalecem fenômenos internos suscetíveis à auto-observação, buscamos extrapolar os aspectos afetivos das atitudes linguísticas da população estudada por meio de um matched-guise e de um questionário, instrumentos que investigam percepções sobre cinco dialetos que são freqüentemente encontrados na universidade. Os resultados mostram diferentes avaliações dos dialetos e uma consideração do dialeto de Bogotá como central, prestigioso e padrão, fortemente associado às formas de oralidade acadêmica.

Palavras-chave: atitudes linguísticas; espanhol padrão; oralidade; dialetos 


\section{Introducción}

Según Alvar (1996), aquello que define el estatus de una variedad determinada de la lengua es su nivel de prestigio. En ese sentido, Colombia en su historia como Estado nación se ha inclinado institucionalmente por una política lingüística tendiente tanto al monolingüismo (en perjuicio de las lenguas indígenas) como a una suerte de centralismo dialectal, entendido como la promoción e identificación de aspectos de corrección y presigio en el dialecto (Von der Walde, 2002). En este sentido, la sociedad colombiana en el ámbito educativo ha propugnado una variedad conservadora del español, lo que ha generado actitudes de discriminación lingüística hacia aquellos aprendientes que provienen de realidades dialectales diferentes a la canónica.

Sin embargo, a finales del siglo pasado y después de la Constitución de 1991 - que significó una ruptura histórica con el Estado confesional, conservador y monolingüe de los cien años anteriores -, el reconocimiento institucional de la sociedad colombiana como un colectivo multiétnico y pluricultural ha permitido la puesta en marcha no solo de nuevas políticas lingüísticas, sino también de dinámicas sociales que propugnan el reconocimiento de lo diverso. De este modo, las universidades colombianas se presentan hoy como un espacio plural en el que se establecen dinámicas más o menos eficaces de comunicación intercultural, normalización y estandarización lingüística (Calvet, 1997).

En este panorama nacional, la ciudad de Cali y su población universitaria se manifiestan como un espacio particularmente diverso desde el punto de vista étnico, cultural y lingüístico. Los flujos migratorios hacia la ciudad de Cali a lo largo del siglo XX explican gran parte de su crecimiento demográfico. Entre 1920 y 1970 se producen las principales olas migratorias provenientes de la región Andina nariñense, compuestas por mestizos y mestizo-indígenas principalmente. Las olas migratorias de población afrodescendiente sucedidas durante todo el siglo XX se constituyen en un factor fundamental para redibujar el mapa demográfico en el área urbana y periférica de Cali (Urrea, 2012).

De esta forma, aparecen en la ciudad poblaciones permanentes de migrantes provenientes de la costa del Pacífico, del norte del Cauca y de la región Andina nariñense. Esta población migrante, perteneciente a otros paradigmas lingüísticos y culturales, actualmente se yergue como un actor social de importancia en diferentes ámbitos sociales, entre ellos el educativo. La Pontificia Universidad Javeriana de Cali es uno de los principales centros educativos del suroccidente colombiano y su comunidad universitaria es representativa de la diversidad cultural y dialectal característica de la 
región. Esta universidad recibe semestralmente alrededor de un $30 \%$ de estudiantes que provienen de otras regiones del país. Tanto por sus características geolectales como por sus usos pragmáticos e interculturales diversos, estos estudiantes pueden encontrar tensiones al integrarse al estándar lingüístico académico oral, entendido este último como un registro elaborado del español altamente intercomprensivo en el marco de la comunidad hispanohablante. Este tipo de dificultades han sido estudiadas desde diferentes perspectivas, entre la cuales sobresale la de Bernstein (1964) acerca de los códigos elaborados y los códigos restringidos, que establece una relación entre fracaso escolar y diferencia de códigos hogar-escuela.

A nuestro juicio, este ámbito requiere particular atención, pues sin la puesta en marcha de estrategias orientadas a mejorar la comunicación intercultural, el proceso académico de los estudiantes puede enfrentar tropiezos. Las investigaciones de Erdösová (2011) en México, acerca del valor identitario de los estudiantes universitarios hablantes de lenguas indígenas, y los estudios de Hernández (2012) respecto a la valoración positiva o negativa de las variedades idiomáticas del país por parte de los estudiantes de la Universidad Nacional de Colombia, demuestran la importancia de las investigaciones sobre las actitudes lingüísticas en los procesos de integración y de comunicación intercultural de estudiantes pertenecientes a otras realidades lingüísticas.

\section{Estudios de actitudes lingüísticas}

Según Moreno (2005), la causa fundamental de la variación de una lengua se relaciona con las creencias y percepciones de sus hablantes, en la medida en que a partir de estas percepciones una comunidad de habla dada otorga determinado valor a ciertos usos lingüísticos, en perjuicio de otros considerados menos prestigiosos. La naturaleza de estas creencias, que constituyen el ámbito de estudio de las actitudes lingüísticas, ha sido objeto de un importante debate teórico desde los años sesenta, momento en el que se inaugura este campo de investigación. A continuación, procuraremos definir, en sus líneas más generales, los tópicos fundamentales del debate teórico alrededor de los estudios de actitudes lingüísticas.

En primer lugar, las actitudes lingüísticas se han considerado como pertenecientes a la esfera de lo psicosocial. Fishman (1988) y Cestero y Paredes (2015) distinguen dos aproximaciones teóricas en ese sentido. Por una parte, la aproximación mentalista, según la cual las actitudes lingüisticas son una disposición mental no observable desde el exterior, que debe ser inferida a partir de un autoexamen del sujeto. Por otra parte, el enfoque conductual, según el cual las actitudes lingüísticas son realidades observables, 
en la medida en que son dependientes de variables externas que producen una actitud lingüística determinada.

Así mismo, se puede señalar que quienes conciben las actitudes lingüísticas como una disposición mental, tienden a considerarlas como una estructura múltiple, mientras quienes las conciben como una conducta meramente externa tienden a catalogarlas como una unidad indivisible (Soler, 1999). Para Lambert y Lambert (1964), Rosenberg (1960) y Rokeach (1968), las actitudes lingüísticas tienen tres componentes: el cognitivo (creencias), el afectivo (emociones y sentimientos) y el comportamental (reacciones y actuaciones frente al objeto).

Particularmente, en este artículo nos situamos desde una aproximación mentalista y centramos la atención en los aspectos afectivos de las actitudes lingüísticas, relacionados con el prestigio social y la valoración que los hablantes hacen de una lengua. Es importante señalar que la mayor parte de los estudios sobre actitudes que se han llevado a cabo se han referido a las situaciones de bilingüismo, plurilingüismo y lenguas en contacto; entre tanto, el estudio respecto a las actitudes de hablantes de variedades de una misma lengua ha recibido menor atención.

En Colombia, los estudios de actitudes se han acercado a aspectos relevantes de la educación formal bilingüe inglés-español (Dávila, 2012; López, Peña \& Guzmán, 2011), pero, según Córdoba (2012), los estudios de actitudes lingüísticas referidos a comunidades bilingües en lengua indígena/español son escasos.

Entre estos últimos hay que resaltar los de Soler (1999), sobre la comunidad inga, y los de Alvar (1996), sobre las comunidades huitoto. En el campo específico de la investigación relativa a actitudes lingüísticas entre hablantes de variedades dialectales de una misma lengua, podríamos nombrar los de Duarte (2005), acerca de las actitudes lingüísticas de los bogotanos respecto a las variedades dialectales en otras regiones, y Salazar (2015) referidos a las actitudes lingüísticas de los monterianos hacia su propio dialecto. Entre tanto, en el ámbito regional, es importante mencionar el estudio realizado por Caicedo (1992), Dialecto y sociedad en Buenaventura, en el que se destaca el prestigio del que goza el geolecto regional, rico en africanismos. Esta conclusión es interesante, dado que en general esta región del país, como el resto de la zona Pacífica, ha sufrido condiciones de marginalidad económica y por lo tanto podría esperarse una actitud diferente de los hablantes respecto a su dialecto.

En ese sentido, este artículo busca describir los aspectos afectivos de las actitudes lingüísticas de 53 estudiantes de diferentes zonas geolectales de Colombia que ingresan 
a la Pontificia Universidad Javeriana de Cali. Consideramos que un estudio de actitudes lingüísticas en el contexto de esta población universitaria es una interesante perspectiva de investigación, que no solo permitirá indagar acerca de las percepciones de los hablantes respecto a los recursos lingüísticos del medio, sino que también conlleva una dinámica que posibilita la puesta en marcha de procesos de autoobservación del propio recurso lingüístico. Este último aspecto probablemente redundará, a su vez, en un mejor monitoreo y una mayor consciencia del proceso personal de aprendizaje.

\section{Materiales y métodos}

Este estudio sigue una perspectiva mentalista, que hace referencia a la indagación por un estado interno o mental del individuo hacia una situación sociolingüística dada (Moreno, 2005). Tomando en cuenta lo anterior, se recurre al autorreporte. Hemos establecido tres dimensiones que nos permitieron extrapolar las percepciones de los hablantes respecto a los usos lingüísticos. Naturalmente, estas dimensiones son posibles a partir de una concepción multicomponencial de las actitudes lingüísticas: 1) prestigio: concebido como el estatus asignado a una variedad lingüística por los individuos pertenecientes a una comunidad de habla; 2) metacognición: percepción de la corrección formal de su dialecto y de los otros dialectos con los que entra en contacto; 3) intercomprensión: dimensión relacionada con el potencial comunicativo del propio dialecto respecto a otras variedades lingüísticas. En particular, nos hemos centrado en este artículo en la dimensión afectiva de las actitudes, correspondiente al componente de prestigio.

\section{Enfoque investigativo}

Esta investigación empleó un enfoque cualitativo, pues buscaba caracterizar las actitudes desde el punto de vista de los participantes. Se utilizaron también estrategias cuantitativas para llegar a una comprensión más completa del fenómeno que se investigó. Cabe recordar que la definición de una investigación como cualitativa o cuantitativa no está dada por las técnicas que se aplican, sino por los presupuestos epistemológicosontológicos y la forma en la que los datos se interpretan (Bonilla \& Rodríguez, 2005; Páramo, 2011). El diseño de investigación fue de corte descriptivo transversal, por cuanto se pretendió caracterizar el fenómeno de estudio en un momento específico, sin la intención de controlar o medir variables.

\section{Participantes}

La investigación se llevó a cabo con 53 estudiantes de primer semestre de la Pontificia Universidad Javeriana de Cali, institución colombiana de educación superior 
de carácter privado que cuenta con alrededor de 8000 estudiantes y 22 programas académicos de pregrado. La convocatoria para participar en la investigación se hizo con estudiantes ingresantes que estaban matriculados en los cursos introductorios de lenguaje. En el primer semestre del 2018 había cerca de 700 estudiantes matriculados, el $30 \%$ de ellos provenientes de otras variedades dialectales. Por las características del tema abordado, se optó por un muestreo por conveniencia (McMillan \& Schumacher, 2005), en el que la participación dependió de la voluntad y disposición de los informantes, sin buscar una representación estadística de la población.

\section{Instrumentos de recolección de información}

En primer lugar, se empleó la técnica del cuestionario matched-guise o prueba de pares ocultos (Lambert, Hodgson, Gardner \& Fillenbaum, 1960), que permite la recolección de datos implícitos de las actitudes a través de estrategias indirectas. Este instrumento prevéla escuchadegrabaciones de un mismo locutor que camuflasu identidad a través del uso de diferentes variedades lingüísticas (en esta investigación, de diferentes variedades geolectales). Los informantes, por su parte, deben escoger entre una serie de binomios ocultos/disfrazados. En este caso utilizamos la escala de diferencial semántico de Osgood, Suci y Tannenbaum (1957) para evaluar las percepciones expresadas en pares de conceptos opuestos relacionados con algunas variedades dialectales colombianas. Los binomios se formularon de acuerdo con diferentes variables que buscan desentrañar las percepciones del informante. Estas variables se refieren a tres esferas: atractivo individual, atractivo social y estatus socioeconómico (Blass Arroyo, 1977; González, 2008). Las variedades dialectales colombianas tomadas en cuenta para el cuestionario matched guise son: bogotano, costeño pacífico, antioqueño, vallecaucano y nariñense (clasificación retomada de Montes, 1996).

El instrumento estuvo integrado por 15 pares, 8 correspondientes a variables cognitivas y 7 a variables afectivas. Estas últimas constituyen el objeto de análisis de este artículo. La prueba inició con la administración de un formulario en línea que contenía los pares, y los participantes debían evaluar su percepción de 5 grabaciones con las variedades dialectales seleccionadas. El instrumento evidenciaba el número de la grabación que los participantes estaban escuchando, la consigna de la actividad, la explicación visual de la escala de diferencial semántico ( 1 a 7 , siendo 1 muy negativo y 7 muy positivo) y posteriormente los binomios que debían evaluarse. La dimensión afectiva incluyó los siguientes pares: 1) mal educado-educado, 2) vulgar-culto, 3) tonto-inteligente, 4) pobre-rico, 5) aburrido-divertido, 6) desagradable-agradable y 7) desconfiable-confiable. 
El segundo instrumento fue un cuestionario que permitió acceder de forma más directa a la información. Este se aplicó después del matched-guise, también a través de un formulario en línea. El instrumento estuvo constituido por 32 preguntas, 24 de ellas de opción múltiple y 8 preguntas abiertas. El cuestionario recogió información demográfica de los participantes (edad, sexo, carrera, estrato socioeconómico, lugar de origen, dialecto con el que se identificaba, tiempo de permanencia en la ciudad de Cali), sus percepciones sobre las relaciones entre las formas de hablar y aspectos como la socialización y la consecución de un trabajo. De igual manera, incluyó preguntas sobre las relaciones entre las formas de hablar de los participantes y las características de la oralidad académica o registros propios del ámbito universitario.

\section{Técnicas de análisis}

Respecto al análisis de los datos, y considerando las intenciones exploratorias del presente trabajo, para analizar los datos cuantitativos se tomó como referente laestadística descriptiva (Hueso \& Cascant, 2012) con la presentación de tablas de frecuencia y porcentajes tanto en las preguntas cerradas del cuestionario como en los resultados del matched-guise. Para las respuestas abiertas del cuestionario se usó la técnica de análisis de contenido, a fin de ordenar, categorizar e interpretar las declaraciones de los informantes. En este análisis, aunque se consideraron algunas categorías a priori basadas en el marco teórico y en el estado de la cuestión, se buscó que las categorías emergieran a partir de los datos (Ballesteros \& Mata, 2014). Para apoyar el proceso de análisis de contenido se usó el software Atlas. Ti 8.0.

\section{Resultados}

A partir del análisis de los datos arrojados en los dos instrumentos de recolección, los resultados se han organizado en cuatro grandes categorías: en primer lugar, se ubican las características demográficas de los participantes; en segundo lugar, las variables de prestigio asociadas a las formas de hablar; en tercer lugar, los aspectos afectivos de las actitudes lingüísticas asociadas a los cinco dialectos evaluados y, finalmente, las actitudes generales hacia el registro estándar del español en el contexto académico.

\section{Características demográficas de los participantes}

Como se indicó, en esta investigación participaron de forma voluntaria 53 estudiantes universitarios de primer año, que estaban cursando distintas carreras en las áreas de ingeniería, humanidades, ciencias sociales, salud y ciencias administrativas. 
El promedio de edad de los participantes fue de 17,7 , en un rango entre 15 y 28 años. 51 \% fueron hombres y $49 \%$ mujeres, todos provenientes de contextos geográficos distintos a la ciudad de Cali y alrededores. Los participantes se identificaron con cerca de 15 variedades dialectales distintas. Los dialectos más frecuentes fueron el nariñense (26\%), el costeño-pacífico (22 \%) y el antioqueño (16\%). Esta mayor frecuencia puede explicarse debido a la cercanía geográfica de los municipios en los que normalmente se encuentran estos dialectos con la ciudad de Cali.

\section{Variables de prestigio asociadas a las formas de hablar}

Esta categoría incluye las relaciones que, en general, los participantes establecen entre las formas de hablar y variables de prestigio como la inteligencia, la profesión, el nivel educativo, el poder económico y la confiabilidad de las personas.

Al respecto, la Figura 1 muestra que la mayoría de los participantes (88\%) considera que la forma de hablar evidencia el nivel educativo de las personas. También con un alto porcentaje, se establece una relación con la inteligencia y el poder económico, mientras que estos nexos no son tan claros en aspectos como la confiabilidad y la identificación de la profesión.

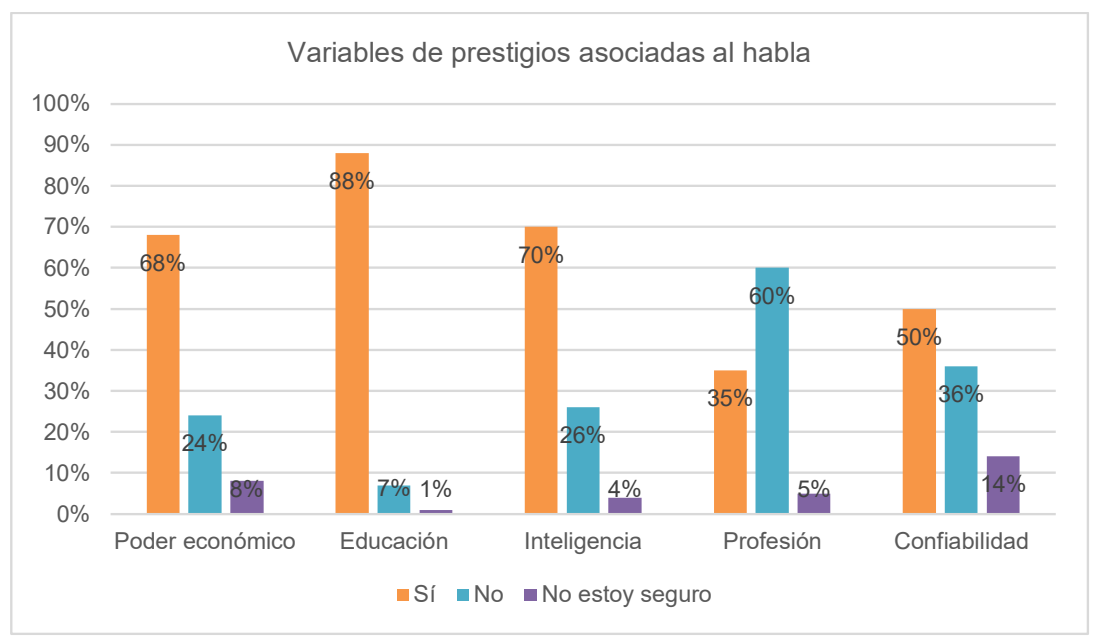

Figura 1. Variables de prestigio asociadas al habla.

Al preguntar a los participantes acerca de la existencia de un dialecto "correcto", el 89 \% considera que sí hay formas más elaboradas de habla y que esto puede tener incidencia en aspectos como la consecución de un trabajo o mayor facilidad en la socialización con otras personas. 
De acuerdo con la Figura 2, en cuanto al trabajo, más de la mitad de los estudiantes indicaron que podría ser más fácil si el dialecto es el bogotano (dialecto central en Colombia), mientras que un $30 \%$ considera que es indiferente.

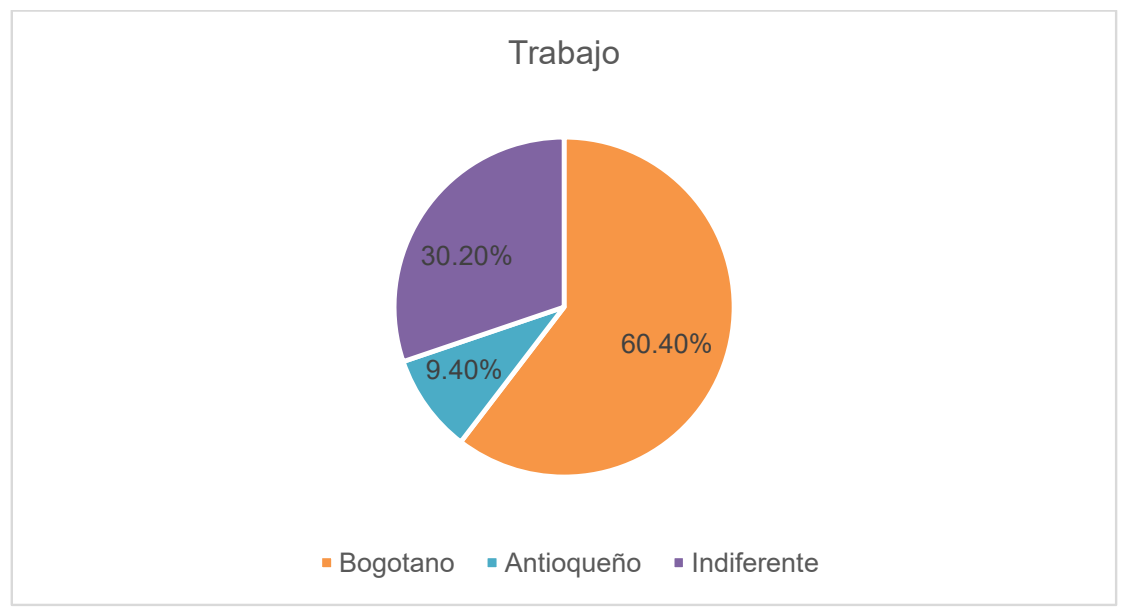

Figura 2. Dialectos que pueden favorecer la consecución de un trabajo.

Por otra parte, se identifica una mayor variedad respecto a los dialectos que pueden favorecer la socialización. Aparece, en primer lugar ( $40 \%$ ) el acento vallecaucano, que es el predominante en la región en la que los estudiantes viven en el momento de la investigación; otro porcentaje considera que es indiferente; aparece también el acento antioqueño y en menor medida el bogotano (Figura 3).

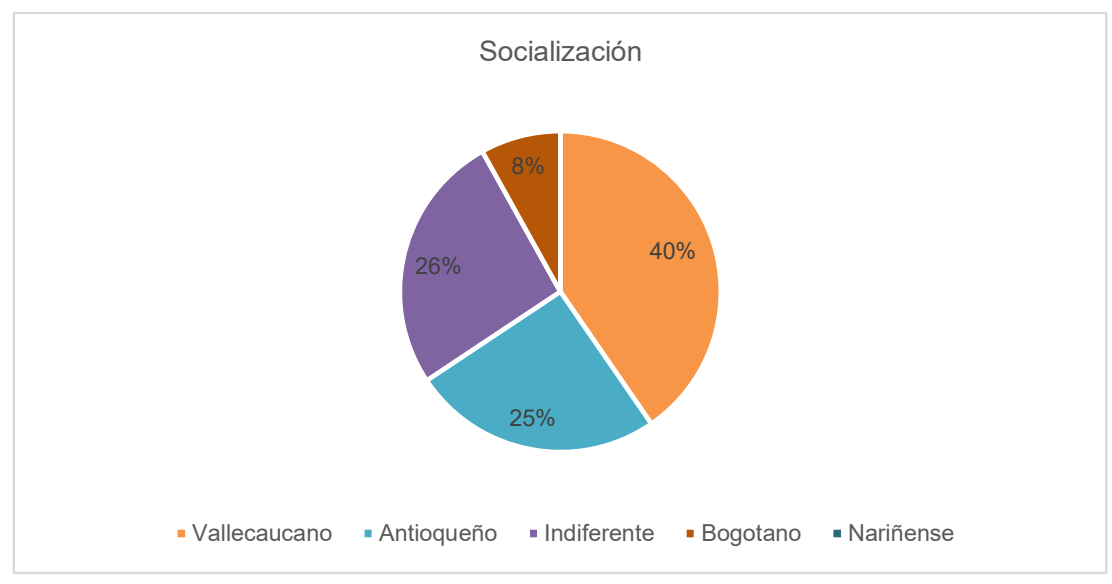

Figura 3. Dialectos que pueden favorecer la socialización. 


\section{Aspectos afectivos de las actitudes lingüísticas hacia los dialectos estudiados}

Esta categoría evidencia la valoración afectiva de los participantes hacia los dialectos estudiados. En el matched-guise, como se indicó en la metodología, se usaron grabaciones de una misma persona que presentó un mismo mensaje variando las características del habla de acuerdo con cinco acentos. En la valoración afectiva se incluyeron siete pares, agrupados en tres características: percepciones sobre el nivel educativo del hablante, percepciones sobre el nivel económico del hablante y características personales atribuidas. Respecto a las percepciones sobre el nivel educativo del hablante, la Tabla 1 evidencia una valoración muy positiva del dialecto central, el bogotano, cuyas puntuaciones se sitúan en los rangos superiores de la escala de diferencial semántico en aspectos como "educado", "culto" e "inteligente". También cuentan con una valoración positiva, aunque en menor medida, los dialectos nariñense y antioqueño, luego el vallecaucano y, en última instancia, el costeño pacífico.

Con sombreado gris se han destacado aquellos niveles de la escala en los que se concentran los porcentajes de respuesta de los informantes.

Tabla 1. Percepciones sobre el nivel educativo del hablante en cada dialecto

\begin{tabular}{|l|l|c|c|c|c|c|c|c|c|}
\hline \multicolumn{7}{|c|}{ Valoración en escala del diferencial semántico } \\
\hline & Negativo & $\mathbf{1}$ & $\mathbf{2}$ & $\mathbf{3}$ & $\mathbf{4}$ & $\mathbf{5}$ & $\mathbf{6}$ & $\mathbf{7}$ & Positivo \\
\hline & Mal educado & & & & & & & Educado \\
\hline Vallecaucano & & $3,8 \%$ & $1,9 \%$ & $3,8 \%$ & $28,3 \%$ & $24,5 \%$ & $30,2 \%$ & $7,5 \%$ & \\
\hline Bogotano & & $0,0 \%$ & $0,0 \%$ & $0,0 \%$ & $0,0 \%$ & $5,7 \%$ & $13,2 \%$ & $81,1 \%$ & \\
\hline Nariñense & & $0,0 \%$ & $0,0 \%$ & $5,7 \%$ & $7,5 \%$ & $20,8 \%$ & $18,9 \%$ & $47,2 \%$ & \\
\hline Antioqueño & & $0,0 \%$ & $1,9 \%$ & $11,3 \%$ & $9,4 \%$ & $30,2 \%$ & $24,5 \%$ & $22,6 \%$ & \\
\hline Costeño-Pacífico & & $0,0 \%$ & $3,8 \%$ & $24,5 \%$ & $22,6 \%$ & $28,3 \%$ & $9,4 \%$ & $1,9 \%$ & \\
\hline & Vulgar & & & & & & & & Culto \\
\hline Vallecaucano & & $1,9 \%$ & $3,8 \%$ & $9,4 \%$ & $20,8 \%$ & $30,2 \%$ & $24,5 \%$ & $9,4 \%$ & \\
\hline Bogotano & & $0,0 \%$ & $0,0 \%$ & $0,0 \%$ & $1,9 \%$ & $3,8 \%$ & $11,3 \%$ & $83,0 \%$ & \\
\hline Nariñense & & $1,9 \%$ & $0,0 \%$ & $5,7 \%$ & $11,3 \%$ & $28,3 \%$ & $15,1 \%$ & $37,7 \%$ & \\
\hline Antioqueño & & $0,0 \%$ & $0,0 \%$ & $11,3 \%$ & $15,1 \%$ & $24,5 \%$ & $30,2 \%$ & $18,9 \%$ & \\
\hline Costeño-Pacífico & & $1,9 \%$ & $1,9 \%$ & $32,1 \%$ & $35,8 \%$ & $17 \%$ & $5,7 \%$ & $5,7 \%$ & \\
\hline & Tonto & & & & & & & & Inteligente \\
\hline Vallecaucano & & $1,9 \%$ & $1,9 \%$ & $3,8 \%$ & $20,8 \%$ & $26,4 \%$ & $43,4 \%$ & $1,9 \%$ & \\
\hline Bogotano & & $0,0 \%$ & $0,0 \%$ & $0,0 \%$ & $0,0 \%$ & $7,5 \%$ & $18,9 \%$ & $73,6 \%$ & \\
\hline
\end{tabular}




\begin{tabular}{|l|c|c|c|c|c|c|c|c|c|}
\hline Nariñense & & $0,0 \%$ & $0,0 \%$ & $7,5 \%$ & $13,2 \%$ & $22,6 \%$ & $22,6 \%$ & $34 \%$ & \\
\hline Antioqueño & & $0,0 \%$ & $0,0 \%$ & $11,3 \%$ & $13,2 \%$ & $24,5 \%$ & $35,8 \%$ & $15,1 \%$ & \\
\hline Costeño-Pacífico & & $0,0 \%$ & $1,9 \%$ & $28,3 \%$ & $26,4 \%$ & $18,9 \%$ & $3,8 \%$ & $18,9 \%$ & \\
\hline
\end{tabular}

Las percepciones sobre el nivel económico del hablante, de acuerdo con cada variación dialectal, tienen un comportamiento similar a los expuestos anteriormente. En este caso, la producción del hablante con dialecto bogotano es percibida como la de mayores posibilidades económicas, seguidas por los dialectos vallecaucano, nariñense y antioqueño, mientras que el dialecto costeño-pacífico tiene una percepción más negativa (Tabla 2).

Tabla 2. Percepciones sobre el nivel económico del hablante en cada dialecto

\begin{tabular}{|l|l|c|c|c|c|c|c|c|c|}
\hline \multicolumn{8}{|c|}{ Valoración en escala del diferencial semántico } \\
\hline & Negativo & $\mathbf{1}$ & $\mathbf{2}$ & $\mathbf{3}$ & $\mathbf{4}$ & $\mathbf{5}$ & $\mathbf{6}$ & $\mathbf{7}$ & \multicolumn{2}{|c|}{ Positivo } \\
\hline & Pobre & & & & & & & & Rico \\
\hline Vallecaucano & & $3,8 \%$ & $1,9 \%$ & $3,8 \%$ & $28,3 \%$ & $34,0 \%$ & $26,4 \%$ & $1,9 \%$ & \\
\hline Bogotano & & $0,0 \%$ & $0,0 \%$ & $0,0 \%$ & $3,8 \%$ & $13,2 \%$ & $15,1 \%$ & $67,9 \%$ & \\
\hline Nariñense & & $0,0 \%$ & $0,0 \%$ & $9,4 \%$ & $22,6 \%$ & $34 \%$ & $22,6 \%$ & $11,3 \%$ & \\
\hline Antioqueño & & $0,0 \%$ & $1,9 \%$ & $9,4 \%$ & $20,8 \%$ & $30,2 \%$ & $26,4 \%$ & $9,4 \%$ & \\
\hline Costeño-Pacífico & & $0,0 \%$ & $11,3 \%$ & $34 \%$ & $34 \%$ & $18,9 \%$ & $0,0 \%$ & $1,9 \%$ & \\
\hline
\end{tabular}

Finalmente, respecto a las características personales atribuidas al hablante de acuerdo con los dialectos, se evidencian mayores variaciones que en las categorías anteriores. En el par aburrido-divertido hay una mayor concentración en los rangos más altos para los dialectos vallecaucano y antioqueño, un porcentaje más distribuido del dialecto bogotano a lo largo de la escala y una valoración media para los acentos nariñense y costeño-pacífico (Tabla 3).

Tabla 3. Percepciones sobre las características personales del hablante en cada dialecto

\begin{tabular}{|l|l|c|c|c|c|c|c|c|c|}
\hline \multicolumn{8}{|c|}{ Valoración en escala del diferencial semántico } \\
\hline & Negativo & $\mathbf{1}$ & $\mathbf{2}$ & $\mathbf{3}$ & $\mathbf{4}$ & $\mathbf{5}$ & $\mathbf{6}$ & $\mathbf{7}$ & Positivo \\
\hline & Aburrido & & & & & & & & Divertido \\
\hline Vallecaucano & & $0,0 \%$ & $1,9 \%$ & $3,8 \%$ & $5,7 \%$ & $24,5 \%$ & $34,0 \%$ & $30,2 \%$ & \\
\hline Bogotano & & $3,8 \%$ & $5,7 \%$ & $11,3 \%$ & $24,5 \%$ & $11,3 \%$ & $13,2 \%$ & $30,8 \%$ & \\
\hline Nariñense & & $3,8 \%$ & $13,2 \%$ & $18,9 \%$ & $20,8 \%$ & $26,4 \%$ & $3,8 \%$ & $13,2 \%$ & \\
\hline Antioqueño & & $0,0 \%$ & $0,0 \%$ & $1,9 \%$ & $9,4 \%$ & $22,6 \%$ & $39,6 \%$ & $26,4 \%$ & \\
\hline Costeño-Pacífico & & $3,8 \%$ & $5,7 \%$ & $26,4 \%$ & $28,3 \%$ & $28,3 \%$ & $1,9 \%$ & $5,7 \%$ & \\
\hline
\end{tabular}


Tabla 3. Continuación

\begin{tabular}{|l|c|c|c|c|c|c|c|c|c|}
\hline & Desagra-dable & & & & & & & & Agradable \\
\hline Vallecaucano & & $1,9 \%$ & $5,7 \%$ & $1,9 \%$ & $11,3 \%$ & $35,8 \%$ & $26,4 \%$ & $17,0 \%$ & \\
\hline Bogotano & & $0,0 \%$ & $1,9 \%$ & $1,9 \%$ & $7,5 \%$ & $24,5 \%$ & $15,1 \%$ & $49,1 \%$ & \\
\hline Nariñense & & $0,0 \%$ & $3,8 \%$ & $7,5 \%$ & $15,1 \%$ & $35,8 \%$ & $20,8 \%$ & $17 \%$ & \\
\hline Antioqueño & & $0,0 \%$ & $0,0 \%$ & $5,7 \%$ & $13,2 \%$ & $18,9 \%$ & $34 \%$ & $28,3 \%$ & \\
\hline Costeño-Paćfico & & $1,9 \%$ & $5,7 \%$ & $17 \%$ & $17 \%$ & $22,6 \%$ & $15,1 \%$ & $20,8 \%$ & \\
\hline & Descon-fiable & & & & & & & & Confiable \\
\hline Vallecaucano & & $0,0 \%$ & $3,8 \%$ & $5,7 \%$ & $15,1 \%$ & $30,2 \%$ & $26,4 \%$ & $18,9 \%$ & \\
\hline Bogotano & & $0,0 \%$ & $0,0 \%$ & $3,8 \%$ & $1,9 \%$ & $3,8 \%$ & $20,8 \%$ & $69,8 \%$ & \\
\hline Nariñense & & $0,0 \%$ & $0,0 \%$ & $9,4 \%$ & $22,6 \%$ & $26,4 \%$ & $17 \%$ & $24,5 \%$ & \\
\hline Antioqueño & & $1,9 \%$ & $1,9 \%$ & $9,4 \%$ & $9,4 \%$ & $20,8 \%$ & $30,2 \%$ & $26,4 \%$ & \\
\hline Costeño-Paćfífo & & $0,0 \%$ & $0,0 \%$ & $13,2 \%$ & $17 \%$ & $11,3 \%$ & $15,1 \%$ & $43,4 \%$ & \\
\hline
\end{tabular}

En los pares desagradable-agradable y desconfiable-confiable se evidenció una percepción más positiva de los cinco dialectos, con porcentajes concentrados en los niveles medios y altos de la escala. Por otra parte, en el cuestionario (instrumento 2) se incluyeron dos preguntas relacionadas específicamente con la valoración afectiva del dialecto vallecaucano, considerando que los estudiantes foráneos se enfrentan principalmente a esta variación dialectal en sus interacciones cotidianas en la ciudad de Cali. Al preguntar a los estudiantes si les gusta la forma de hablar de los vallecaucanos, el 64 \% respondió afirmativamente, 15 \% de forma negativa y 21 \% indicó no estar seguro.

De igual modo, en una pregunta abierta se pidió a los estudiantes que escribieran dos palabras que les permitieran describir el dialecto vallecaucano. Entre estas palabras apareció un grupo relacionado con la poca formalidad o cuidado de los hablantes (confianzudo, descomplicado, descuidado, descortés) constituido por 52 palabras, y otro grupo en que se evidenciaron características con valor positivo como la tranquilidad, la frescura y la amabilidad, constituido por 43 adjetivos (Tabla 4).

Tabla 4. Adjetivos valorativos relacionados con el dialecto vallecaucano. Pregunta abierta.

\begin{tabular}{cccc} 
Adjetivos con valoración negativa & Frecuencia & Adjetivos con valoración positiva & Frecuencia \\
\hline Confianzudo & 17 & Amigable & 9 \\
\hline Descomplicado & 15 & Tranquilo & 9 \\
\hline Descuidado & 9 & Relajado & 7 \\
\hline Descortés & 6 & Gracioso & 5 \\
\hline Vulgar & 3 & Fresco & 5 \\
\hline Grosero & 2 & Confiable & 4 \\
\hline & & Chévere & 4
\end{tabular}




\section{Actitud hacia el español académico}

Esta categoría da cuenta de las percepciones de los participantes hacia las formas de hablar que se usan con frecuencia en las interacciones más formales del contexto académico, lo que hemos denominado de forma general como "registro estándar" o "español académico". En una pregunta del cuestionario se indagó por la afinidad que los estudiantes percibían entre las formas de hablar en la universidad y sus propias formas de hablar (referidas a sus dialectos). La Figura 4 evidencia que cerca de la mitad de los estudiantes identifica afinidad entre estas formas, pero un $38 \%$ encuentra diferencias importantes y el $15 \%$ no está seguro.

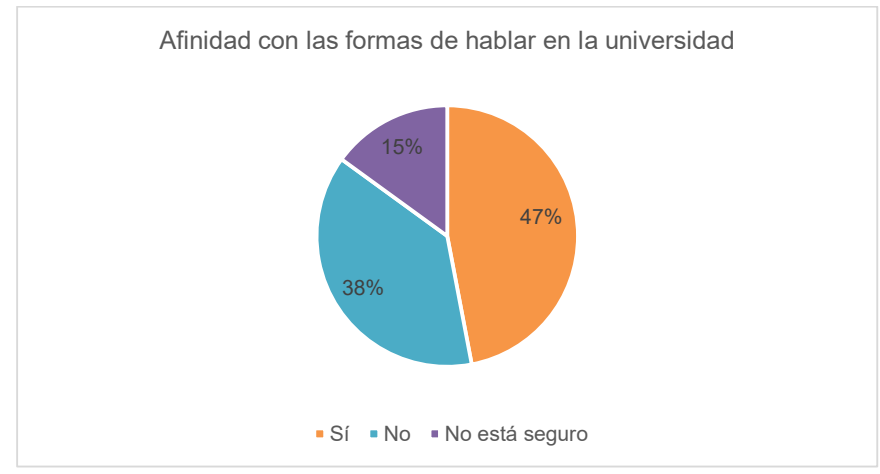

Figura 4. Percepción de la afinidad entre las formas de hablar en la universidad y el propio dialecto de los participantes.

En otra pregunta relacionada con los aspectos afectivos, se indagó con los participantes si habían sentido rechazo de algún miembro de la comunidad universitaria por su forma de hablar. En este caso, se encuentra que más de la mitad no han percibido rechazos en este aspecto, un 20 \% sí y un 15 \% no está seguro (Figura 5).

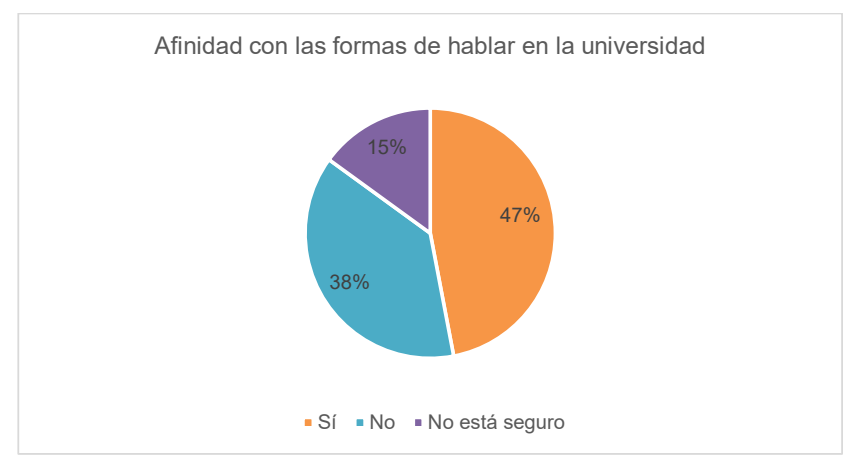

Figura 5. Percepción de rechazo en la universidad relacionado con la forma de hablar de los participantes. 
En las preguntas abiertas se pudieron identificar algunas declaraciones de los participantes respecto a los posibles rechazos o burlas de otros miembros de la comunidad universitaria, relacionados con las formas de hablar. Esto se evidenció con dos grupos principalmente: los nariñenses, quienes han recibido comentarios por sus formas de vocalización o por su entonación, lo que los lleva a preferir participar poco en los espacios de clase; y el grupo de los costeño-pacíficos, predominantemente afros, que también identifican burlas y reproches relacionados con su ritmo de habla (que consideran más lento que el de los otros dialectos) y la ausencia de pronunciación o aspiración de ciertos fonemas. A continuación, se evidencian algunos fragmentos de las respuestas abiertas de los participantes. La letra "E" se refiere a "Estudiante" y el número que le sigue corresponde al número del informante que respondió el cuestionario:

E2: Uno de mis problemas es tener que vocalizar mejor, ya que de donde vengo no se acostumbra a prestar mucha atención a la vocalización y hay ciertos sonidos que no pronunciamos, como la "s" (costeño-pacífico).

E18: Los compañeros se burlan mucho cuando a los nariñenses se nos sale alguna palabra que ellos consideran rara o porque la entonación es diferente. Muchos nariñenses o nariñenses preferimos no bablar tanto en clase para evitar critica (nariñense).

E23: Hay palabras que no sé pronunciar bien y sobre todo en inglés es muy difícil porque me "como" las letras (costeño-pacífico).

E25: A los maestros les falta paciencia para entender que los negros hablamos más suave, que no concretamos las cosas tan rápido, nos tomamos nuestro tiempo bablando, nuestra forma de hablar es distinta, más relajada (costeño-pacífico).

E37: Los compañeros son un poco inmaduros y se burlan de otros acentos, por eso a veces es mejor no participar tanto o aprender a aguantar los chistes (nariñense).

E52: Hablo poco para evitar burlas respecto a mi acento o palabras muy específicas de mi ciudad (nariñense).

\section{Discusión}

Los hallazgos de esta investigación coinciden con las observaciones de Von der Walde (2002) respecto a los aspectos de corrección y prestigio históricamente asociados con la variante bogotana del español colombiano. En esa medida, se puede observar que aún hoy persisten en las actitudes lingüísticas de los estudiantes universitarios de la Pontificia Universidad Javeriana de Cali los estereotipos que se generaron a partir de 
las políticas lingüísticas de normalización y normativización impulsadas por el Estado colombiano desde finales del siglo XIX hasta mediados del siglo XX. En esta medida, los resultados evidencian que el grupo de participantes tiene actitudes lingüísticas positivas hacia los rasgos del dialecto bogotano, asociándolo con un mayor prestigio personal, social y socioeconómico. Esta valoración más positiva del dialecto central genera que sea percibido como deseable en la consecución de un trabajo y también más cercano a las formas de comunicación propias del ámbito universitario, en el que se usa un registro más estándar del español.

En aspectos como la socialización, los dialectos vallecaucano y antioqueño tienen una valoración más positiva. Esto puede deberse a la asociación entre estos dialectos y características positivas de personalidad, como una mayor apertura, tranquilidad 0 comportamientos amistosos. También se puede inferir que las citadas preferencias se relacionan con el papel que estas regiones colombianas juegan en la identidad nacional. Es notable como el Valle del Cauca y los territorios de los departamentos de Caldas, Risaralda, Quindío y Antioquia, corresponden a zonas de notable crecimiento económico y albergan dos de las ciudades más importantes del país (Cali y Medellín). Los dialectos nariñense y costeño-pacífico son los de menor valoración positiva, sobre todo el último, lo que se constituye en un aspecto preocupante, debido a que la mayoría de estudiantes foráneos de la institución provienen de zonas en las que predominan estos dialectos.

A este respecto, es interesante observar que los hallazgos de esta investigación podrían complementar los de Caicedo (1992) respecto a la percepción positiva que tienen los hablantes del dialecto costeño-pacífico de Buenaventura respecto a sus propios usos lingüísticos. Se evidenciaría que esta aceptación autóctona hacia la variante geolectal de esta zona del país, que característicamente presenta rasgos lingüísticos de las lenguas afrohispanoamericanas en diferentes niveles, no es compartida por los informantes de este estudio hablantes de otros dialectos, que la consideran marcada y poco prestigiosa.

Continuando con la reflexión acerca de la menor aceptación afectiva del nariñense y del costeño-pacífico, son precisamente los estudiantes de estos dos grupos quienes han percibido algún tipo de rechazo por sus formas de hablar y, de forma explícita, algunos afirman que para evitar las sanciones sociales prefieren tener una menor participación en los espacios de socialización académica como en los debates en clase. Se evidencia entonces que esta población estudiantil, tanto por sus características geolectales como por sus usos pragmáticos e interculturales diversos, puede presentar dificultades para integrarse al estándar lingüístico académico oral, entendido este último como un registro elaborado del español. El fracaso escolar y las diferencias de códigos hogar-escuela han 
sido estudiados también desde otras perspectivas, como la planteada por Bernstein (1964), en la que se establecen contrastes entre los códigos elaborados y los códigos restringidos. Las implicaciones de estas diferencias pueden ser muy negativas, debido a que pueden afectar tanto el desempeño académico como las posibilidades de construir conocimientos más sólidos a partir del aprendizaje colaborativo con los pares y profesores.

A partir del estudio de la dimensión afectiva de las actitudes lingüísticas hacia los cinco dialectos seleccionados por su importancia en el contexto social de la investigación, se lograron identificar tres grandes desafíos a los que se enfrentan los estudiantes. Por un lado, la aceptación y valoración de su propio dialecto. Esto se evidencia particularmente con los hablantes del dialecto costeño-pacífico, quienes perciben de forma menos positiva las características de sus formas de comunicarse en comparación con los demás dialectos, lo cual es reforzado por la valoración de las personas con las que interactúan cotidianamente y en ámbitos académicos. Un segundo desafío es la sensibilización y comprensión de los hablantes respecto a las variaciones discursivas y culturales existentes en nuestro país. Finalmente, el ajuste a las demandas orales propias del contexto académico, donde se exige un registro más formal y una adecuación a la comunicación con un amplio grupo de interlocutores (Carlino, 2005; Gutiérrez, 2014), sin que esto implique que se reste valor a la diversidad característica de un país multirracial y multicultural como Colombia. Es entonces necesaria la generación de espacios académicos más plurales, en los que se reflexione acerca del valor de las lenguas y de la diversidad de las maneras de comunicación. De igual modo, se requiere de apoyos institucionales que involucren el trabajo con docentes y estudiantes, así como la creación de recursos educativos que fortalezcan aspectos de oralidad académica.

Esta investigación de tipo exploratorio constituye una aproximación al estado de la cuestión en un grupo particular de estudiantes, considerando sus propias percepciones y valoraciones. Estudios con poblaciones más amplias, con otros instrumentos de naturaleza cualitativa como entrevistas (consideradas en la segunda fase de esta investigación), observaciones y análisis de interacciones pueden dar más orientaciones respecto a los modos de potenciar la oralidad académica a partir del aprovechamiento de la diversidad lingüística y cultural existente en el país.

\section{Referencias}

Alvar, M. (1996). Manual de dialectología hispánica. El español de España. El español de América. Madrid: Ariel.

Ballesteros, B. \& Mata, P. (2014). Investigación cualitativa en educación. Madrid: Editorial de la Universidad Nacional de Educación a Distancia. 
Bernstein, B. (1964). Elaborated and Restricted Codes: Their Social Origins and Some Consequences. American Anthropologist, 66(6), 55-69.

Blass Arroyo, J. (1977). Dimensiones sociolingüísticas del cambio de código. A propósito de un corpus radiofónico. ITL Review of Applied Linguistics, (117-118): 117-150.

Bonilla, E. \& Rodríguez, P. (2005). Más allá del dilema de los métodos, la investigación en ciencias sociales. Bogotá: Norma.

Caicedo, H. (1992). Dialecto y sociedad en Buenaventura. Revista Lenguaje, (19-20), 55-67.

Calvet, L. (1997). Las políticas lingüísticas. Buenos Aires: Edicial.

Carlino, P. (2005). Escribir, leer y aprender en la universidad: una introducción a la alfabetización académica. Buenos Aires: Fondo de Cultura Económica.

Cestero, A. \& Paredes, F. (2015). Creencias y actitudes hacia las variedades del español en el siglo XXI: avance de un proyecto de investigación. En Alfal 50 años. Contribuiçoes para os estudos lingüísticos e filológicos. Joao Pessoa, Brasil: Adaltech-ALFAL.

Córdoba, G. (2012). La importancia sobre las actitudes y las valoraciones lingüísticas. Revista Lenguas en Contacto y Bilingüismo, 4, 1-16.

Dávila, D. (2012). Capital lingüístico en inglés y desigualdad de oportunidades educativas: Exploración en dos colegios oficiales de Bogotá. (Tesis de pregrado). Universidad Nacional de Colombia, Bogotá.

Duarte, G. (2005). Actitudes idiomáticas de los bogotanos frente a los dialectos del español de Colombia. En M. Lozano (ed.), Homenaje a José Joaquín Montes Giraldo: estudios de dialectología, lexicografía, lingüística general, etnolingüistica e bistoria cultura. Bogotá: Instituto Caro y Cuervo.

Erdösová, Z. (2011). Las actitudes lingüísticas de los estudiantes de dos modelos educativos: el caso de la Universidad Autónoma del Estado de México (UAEM) y la Universidad Intercultural del Estado de México (UIEM). En Memorias de las 1. ${ }^{\text {as }}$ Jornadas de Lenguas en Contacto (UAN 2011).

Fishman, J. (1988). Sociología del lenguaje. Madrid: Cátedra.

González, J. (2008). Metodología para el estudio de las actitudes lingüísticas. En Actas del XXXVII Simposio Internacional de la Sociedad Española de Lingüística (SEL). Departamento de Lingüística Hispánica y Lenguas Modernas. Pamplona, Servicio de Publicaciones de la Universidad de Navarra.

Gutiérrez, Y. (2014). Concepciones y prácticas sobre la oralidad en la educación media colombiana. Colección tesis doctoral. Bogotá: Comité Editorial Interinstitucional CAIDEUniversidad Distrital Francisco José de Caldas.

Hernández,A. (2012). Actitudes idiomáticas de los estudiantes de la Universidad Nacional de Bogotá ante lenguas indígenas y el español. En Portal de lenguas de Colombia, diversidad y contacto. Bogotá: Instituto Caro y Cuervo. Recuperado de http://www.lenguasdecolombia. gov.co/sites/lenguasdecolombia.gov.co/files/actitudes\%20idiomaticas_0.pdf 
Hueso, A. \& Cascant, M. (2012). Cuadernos docentes en desarrollo. Valencia, España: Universitat Politécnica de Valencia.

Lambert, W. \& Lambert, W. (1964). Chapter 4. In W. Lambert \& W. Lambert (eds.), Social Psychology (pp. 49-69). New Jersey: Englewood Cliffs.

Lambert, W., Hodgson, R., Gardner, R. \& Fillenbaum, S. (1960). Evaluational Reactions to Spoken Language. Journal of Abnormal and Social Psychology, 60(1), 44-51.

López, A., Peña, B. \& Guzmán, M. (2011). Transición hacia el bilingüismo en colegios públicos colombianos. En Bilingüismo en el contexto colombiano: iniciativas y perspectivas en el siglo XXI (pp. 23-45). Bogotá: Uniandes.

McMillan, J. \& Schumacher, S. (2005). Investigación educativa. (5.a ed.). Estados Unidos: Pearson.

Montes, J. (1996). Colombia. En M. Alvar (ed.), Manual de dialectología hispánica. El español de América (pp. 134-145). Madrid: Ariel.

Moreno, F. (2005). Principios de sociolingüística y sociología del lenguaje (2. ${ }^{a}$ ed.). Barcelona: Ariel.

Osgood, C., Suci, G.J. \& Tannenbaum, P.H. (1957). The Measurement of Meaning. Urbana: University of Illinois Press.

Páramo, P. (2011). La falsa dicotomía entre investigación cuantitativa y cualitativa. La investigación en ciencias sociales: estrategias de investigación. Bogotá: Universidad Piloto de Colombia.

Rokeach, M. (1968). The Nature of Attitudes. International Encyclopedia of Social Sciences. New York: MacMillan.

Rosenberg, M. (1960). A Structural Theory of Attitude Dynamics. Public Opinion Quarterly, 24, 319-40.

Salazar, A. (2015). El prestigio frente a la identidad: las actitudes lingüísticas de los monterianos hacia el español hablado en Montería. Cuadernos de Lingüística Hispánica, (25), 39-55.

Soler, S. (1999). Conductas y actitudes lingüísticas de la comunidad indígena inga. Thesaurus: Boletín del Instituto Caro y Cuervo, (54), 911-979.

Urrea, F. (2012). Transformaciones sociodemográficas y grupos sociorraciales en Cali a lo largo del siglo XX y comienzos del siglo XXI. Historia de Cali, siglo XX. Vol 1. Cali, Colombia: Programa Editorial Facultad de Humanidades de la Universidad del Valle.

Von der Walde, E. (2002). Lenguaje y poder: el proyecto de nación en Colombia a finales del siglo XIX. Estudios de Lingüística en España, (16). 